\title{
Divergence and convergence in early embryonic stages of metazoans
}

\author{
Frietson Galis ${ }^{1} \&$ Barry Sinervo ${ }^{2}$ \\ ${ }^{I}$ Institute of Evolutionary and Ecological Sciences, Leiden University, P.O. Box 9516, 2300 RA Leiden, \\ Netherlands, galis@rulsfb.leidenuniv.nl; ${ }^{2}$ Biology Department, University of California Santa Cruz, \\ sinervo@biology.ucsc.edu.
}

Keywords: evolutionary conservation, pleiotropy, cleavage, gastrulation, organogenesis, multicellularity, phylotypic stage

\begin{abstract}
The early stages of organogenesis in metazoans differ drastically between higher order taxa such as phyla and classes. The segmented germ band stage in insects, the nauplius stage of crustaceans, and the neurula/pharyngula stage in vertebrates are examples of this diversification. In striking contrast with this divergence, is the similarity of these stages within these taxa, i.e., within insects, crustaceans, and vertebrates. The early stages of organogenesis, or phylotypic stages, have, thus, remained very similar in most species since the evolutionary origin of the taxa. These phylotypic stages are considerably more similar to each other than to the earlier stages of cleavage and gastrulation. Cleavage and gastrulation stages display not only great variability, but also striking examples of apparent convergence among species in different phyla, for example in the many cases of epiblastic cleavage in yolk-rich eggs. This leads to the paradoxical situation that the overall similarity of cleavage and gastrulation stages is in general higher among metazoans than of the early stages of organogenesis, but within phyla and classes the situation is the reverse. We discuss data on cleavage, gastrulation, and early organogenesis and evaluate possible causes for conservation, homoplasy, and diversification in an attempt to throw light on this paradoxical situation. In addition, we discuss a hypothesis that has been proposed to explain the diversity of early stages of organogenesis at the level of metazoans and the similarity within many phyla and classes.
\end{abstract}

\section{Contents}

Introduction

Similarity due to conservation or homoplasy

Conservation, convergence and divergence in patterns of cleavage

Mechanistic causes of cleavage patterns

Understanding of diversity
Conservation, convergence and divergence in patterns of gastrulation

Conservation, convergence and divergence in early organogenesis

What câuses conservation of early stages of organogenesisrelative to the divergence seen at other stages

Modularity and the divergence of form in late organogenesis

The relationship between divergence of early and late ontogenetic stages

Early diversification foloowed by stasis of early stages of organogenesis

Conclusion

Acknowledgements

References

112

112

\section{Introduction}

The early stages of cleavage and gastrulation are often. remarkably similar among metazoans (Gilbert and Raunio, 1997; fig. 1). The succeeding stage, organogenesis, is far more diverse among metazoans than cleavage and gastrulation (Gilbert and Raunio, 1997). Paradoxically, embryologists have noticed for a long time that within most higher order taxa, e.g. phylum or class, early organogenesis is considerably less variable than earlier stages (Seidel, 1960; Anderson, 1973; Sander, 1983; Hall, 1996; Elinson, 1987). In particular when we judge similarity relative to the number of options available for the stage under consideration. Towards the end of gastrulation relatively diverse ontogenetic patterns converge to a highly similar stage. Examples 
of such highly similar stages during early organogenesis are: the extended/segmented germ-band stage of insects, the nauplius stage of crustaceans, and the neurula/pharyngula stage of vertebrates (Seidel, 1960, Anderson, 1973; Sander, 1983; Elinson, 1987; Hall, 1996; Grbič, 2000; Dahms, 2000; Galis et al., 2002). During cleavage and gastrulation the overall similarity among metazoans is, thus, greater than during early organogenesis, but, within many higher order taxa the reverse is the case and similarity is higher during early organogenesis than during cleavage and gastrulation. For instance, the rapid divisions during cleavage of polyembryonic insects and mammals resemble each other more than the segmented germband stage and the neurula/ pharyngula stage, respectively, i.e., the early organogenesis stages (see below). On the other hand, within mammals phylotypic stages of different species are more similar than cleavage and gastrulation stages and the same holds for the segmented germ band stage in insects (see below). To understand the evolutionary origin of this counterintuitive situation it is necessary to know whether similarity is the result of conservation, convergence, or parallel evolution.

\section{Similarity due to conservation or homoplasy}

It is usually assumed that similarity among early embryonic stages is the result of evolutionary conservation (Seidel, 1960; Sander, 1983; Buss, 1987; Raff, 1996; Hall, 1996). Even though von Baer (1828) did not believe in evolution by descent, he was the first to propose that early changes in ontogeny were more likely to have cascading consequences than later changes when most of development has already taken place. Although this evolutionary constraint is undoubtedly real (Buss 1987), its importance has recently been challenged by the revelation of considerable variation in early embryonic stages. In addition, evolutionary conservation of early embryonic stages may be less strong than previously thought because of similarity due to convergent or parallel evolution (homoplasy). Similarity is almost unavoidable in the early ontogenetic stages of metazoans because of the complete reset that occurs at the initial single- celled stage. Only a limited number of permutations are possible in embryos with a few undifferentiated cells. In contrast, the diversity of multicellular clonal propagules, which lack such a reset to a singlecelled stage, is indeed remarkably high.

Similarity arises because of either similar selection pressures and/or stringent constraints. In the case of constraints, conservation of morphological patterns arises either because no genetic variation is produced, or because invariably negative pleiotropic effects are associated with changes. Similarity in a phylogenetic context, thus, can have four causes:

1) plesiomorphy, shared ancestral traits (inherited similarity evolved once);

2) synapomorphy, shared derived traits (novel similarity evolved once);

3) homoplasy due to common adaptational pressures, or

4) homoplasy due to developmental constraints (convergent derived adaptive traits promoting trait correlation).

\section{Conservation, convergence and divergence in patterns of cleavage}

\section{Mechanistic causes of cleavage patterns}

During cleavage, the zygote rapidly divides into many smaller cells. Patterns of cleavage are determined by a small number of mechanistic factors, variation in which allows a high diversity of cleavage patterns. One mechanistic factor is the amount and distribution of vitelloprotein within the zygotic cytoplasm (Gilbert, 2000). Yolk impedes cleavage. In zygotes with little yolk, cleavage usually separates the embryo into distinct cells (holoblastic cleavage, as in sponges, sea urchins, lancelets, most amphibians and mammals). In zygotes with a large yolk component, cleavage only occurs in the part of the cytoplasm with little or no yolk. Cleavage proceeds only in this part (meroblastic cleavage), leading in cephalopods, fishes, reptiles and birds to a germinal disc on the surface of a large yolk mass (epiblastic cleavage, Figs. 1a and b), and in some crustaceans and some insects to a superficial layer of embryonal cells with yolk in the center (periblastic cleavage). For example, most amphib- 


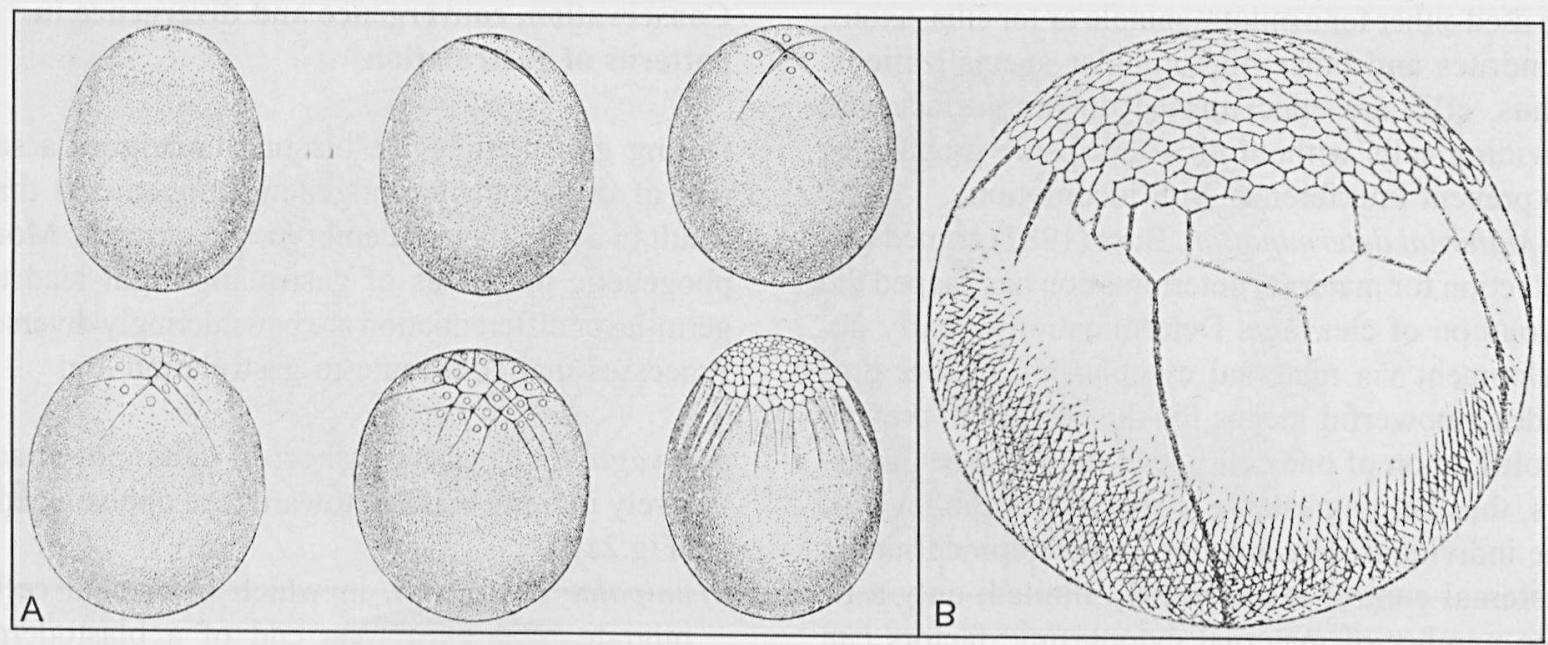

Fig. 1. Convergence. Meroblastic, epiblastic cleavage in (A) the cephalopod Loligo pelalei (after Claus and Grobben, 1917) and (B) in the longnose gar Lepisosteus osseus (after Balfour, 1881).

ians exhibit holoblastic cleavage. However, eleutherodactyline frogs with derived yolky eggs also have secondarily derived epiblastic cleavage (Elinson, 1987). Given the adaptive value of high yolk volume for progeny survival and the extreme convergence of epiblastic or periblastic cleavage among diverse taxa, cleavage mechanisms appear, thus, constrained to evolve as a result of selection for nutrient rich eggs (cause 4, above).

The second mechanism is the angle and timing of mitotic spindle formation, and thus, the orientation of cleaving cells: spiral (various protostomes), radial (various deuterostomes), and rotational (mammals) cleavage.

The third mechanism is differential cell adhesion. Differential adhesion can cause important shape differences, for instance in compaction in mammals and polyembryonic wasps, in which blastomeres are not loosely packed but very closely joined together; or in coeloblastulas, in which the blastula is a hollow ball of cells instead of a solid ball as in stereoblastulae.

\section{Understanding of diversity and similarity}

Diversity in cleavage patterns can be understood in terms of adaptations for embryonic life such as nutrient uptake, locomotion, and maternal determination.
Nutrients can be obtained from either yolk, maternal tissues, or the environment. Specializations for embryonic nutrition uptake can arise as a direct consequence of constraints, e.g. yolk constraints (see above), or as adaptations to specialized conditions. For example, evolution of mammalian viviparity has led to compaction, a conspicuous specialization in which loosely arranged blastomeres suddenly huddle together and form a compact mass (Gilbert, 2000). Compaction and associated intracellular changes function during embryonic implantation in the uterus. Compaction is involved in the separation of the inner cell mass (from which the embryo will develop) from the trophoblast (which provides fetal contributions to the placenta). Interestingly, polyembryonic insects have independently evolved compaction, together with early separation of embryonic and extraembryonic cell lineages (Grbič et al., 1998), presumably because endoparasitism imposes similar spatial constraints as viviparity (an example of cause 3 above).

Locomotor demands also influence cleavage (Buss, 1987). Free-living dispersal stages have external ciliated cells and internal dividing cells. This configuration appears to be largely the result of locomotor demands combined with a universal metazoan constraint (an example of cause 1) - cells cannot divide when ciliated (Buss 1987). Metazoan cells have only one microtubule center, which can 
be used either for a mitotic spindle or for cilia, axons, dendrites and other microtubular specializations. Thus, cilia are concentrated on the surface and dividing cells without cilia are inside propagules to prevent interference with locomotion.

Maternal determination. Buss (1987) argued that selection for maternal determination has shaped the evolution of cleavage. Determination of early development via maternal cytoplasmic factors provides a powerful means for the mother to prevent proliferation of one cell line at the expense of others, thus helping establish selection at the level of the individual. The amount of development under maternal control is necessarily limited; only a finite number of maternal cytoplasmic factors can be provided to the zygote. Unequal cleavage potentially increases effectiveness of maternal control. In unequal cleavage, some cells differentiate and begin zygotic transcription (end of maternal control) and thus lose the capacity to become germ cells, whereas others continue as undifferentiated and divide under maternal control. Differentiated cells usually lose the capacity to divide, which is why they can no longer become germ cells. Unequal cleavage typically occurs in taxa with spiral cleavage such as turbellarians, annelids and molluscs (Buss, 1987).

Polyembryony. Cleavage is radically different in polyembryonic parasitic wasps relative to other insects (Grbi et al., 1998). Cleavage is holoblastic, and the zygote produces not one, but many small embryos. Cellularization occurs immediately, whereas in most other insects (including drosophilids) nuclei first divide into a syncytium and cellularization occurs later. Further specializations of compaction and early separation of embryonic and extraembryonic tissues (see above) are probably caused by the endoparasitic lifestyle, which is to a certain extent convergent with viviparity in mammals, i.e., living in another organism.

In conclusion, cleavage patterns are diverse, but important similarities occur because of evolutionary convergence due to similar embryonic adaptations and constraints. The most striking examples of convergent embryonic adaptations are compaction in mammals and polyembryonic insects and epiblastic cleavage in yolk-rích embryos.

\section{Conservation, convergence and divergence in patterns of gastrulation}

During gastrulation, the blastula undergoes a series of dramatic morphogenetic movements that result in a multilayered embryo, the gastrula. Morphogenetic processes of gastrulation that lead to germ-layer differentiation are bewilderingly diverse. Processes that contribute to gastrulation are:

a) invagination, where a sheet of cells moves actively into the blastula towards the opposite side (Fig.2a);

b) unipolar ingression, in which individual cells migrate inwards at one end of a blastoderm (Fig.2a);

c) multipolar ingression, in which individual cells migrate inwards from all points of the blastocoel;

d) epiboly, the migration of apical cells over the other blastula cells, thus forming an external layer of cells (Fig. 2b);

e) involution, the moving in of a layer of cells so that it spreads over the inner side of the outer layer of cells (Fig. 2b);

f) primary delamination, in which the cells of a blastoderm divide such that one daughter cell remains at the surface and the other one moves to the interior (Fig. 2c); and

g) secondary delamination, in which a solid tissue of cells segregates into two layers. Usually combinations of these processes form part of gastrulation, e.g. invagination and multipolar ingression (Fig. 2a), involution and epiboly (Fíg. 2b).

The combination of possibilities has led to a wide variety of morphogenetic processes between and within phyla and other higher taxa. Cnidarians, for example, exhibit seven different types of gastrulation (Gilbert and Raunio, 1997). In insects, there are drastic differences in gastrulation between short, intermediate, and long-germ band insects, polyembryonic wasps being even more derived. Within teleosts and mammals, gastrulation is highly variable (Collazo et al., 1994; Viehbahn, 1999); for example, the embryonic disc of rodents is cupshaped instead of flat, and germ layers are inverted 


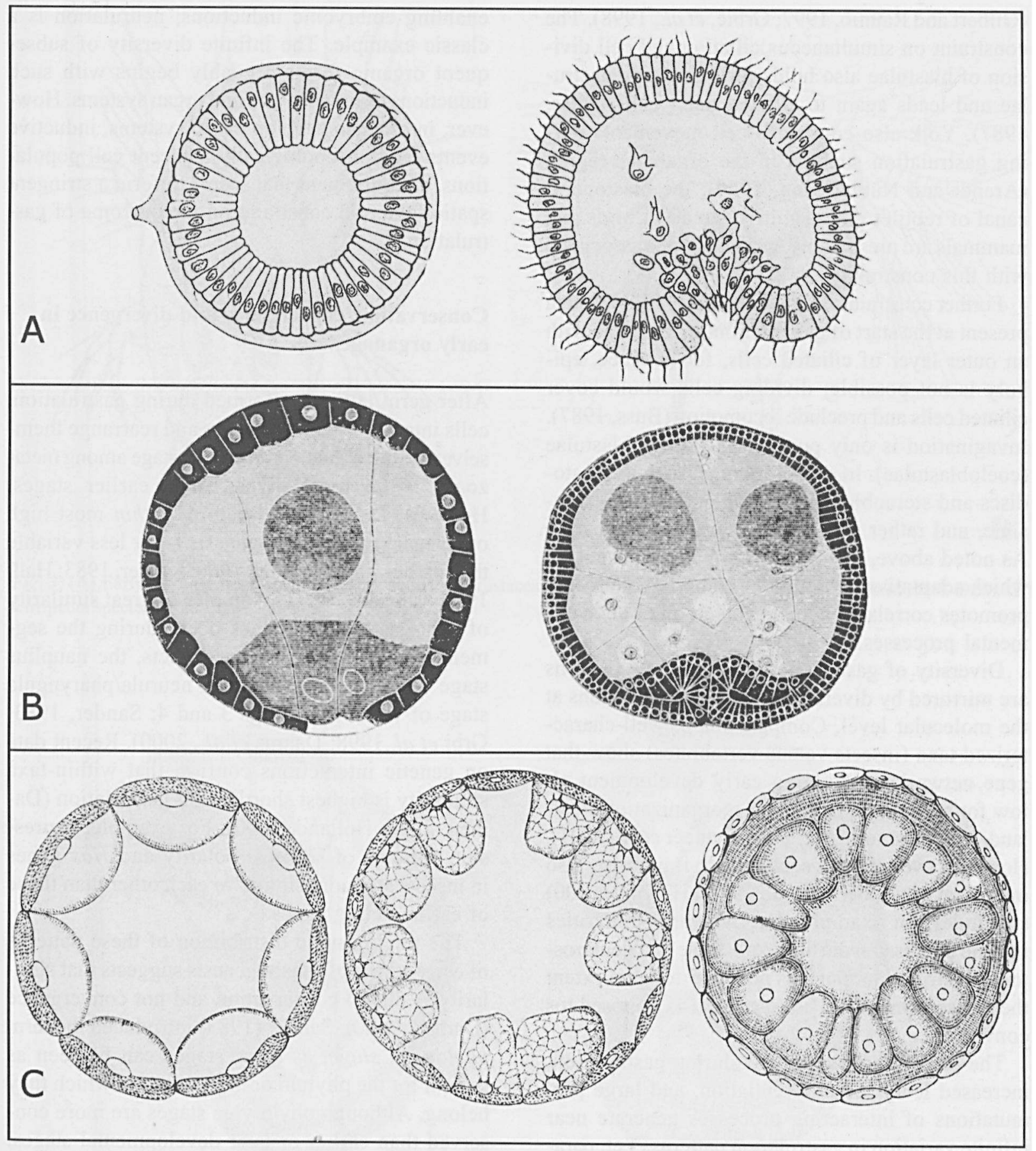

Fig. 2. (A) Diversity of gastrulation. Invagination and unipolar ingression during gastrulation in the echinoderm Holothuria tubulosa (after Balfour 1881). (B) Epiboly and involution in the echiuran Bonellia viridis (after Spengel in Balfour 1881). (C) Primary delamination in the cnidarian Geryona spec. (after Claus and Grobben, 1917).

with endoderm outside and ectoderm inside.

Gastrulation is diverse but also characterized by similarity due to similar constraints and selection pressures. The important selection pressures are, as in cleavage, mainly related to locomotion and nutrient uptake. Viviparous and oviparous gastrulating embryos have numerous adaptations relevant toward living in eggs and other maternal tissues 
(Gilbert and Raunio, 1997; Grbič, et al., 1998). The constraint on simultaneous ciliation and cell division of blastulae also holds for free-living gastrulae and leads again to ciliated outer cells (Buss, 1987). Yolk also constrains cell movements during gastrulation such as in the organizer region (Arendt and Nübler-Jung, 1999); the blastoporal canal of reptiles and primitive streak of birds and mammals are presumably specializations associated with this constraint.

Further constraints follow from the type of blastula present at the start of gastrulation. In blastulae with an outer layer of ciliated cells, for instance, epiboly is not possible; dividing cells would cover ciliated cells and preclude locomotion (Buss, 1987). Invagination is only possible in hollow blastulae (coeloblastulae). In solid blastulae, such as blastodiscs and stereoblastulae, invagination is not possible, and rather epiboly plays an important role. As noted above, this is an example of cause 4 , in which adaptative evolution of embryonic nutrition promotes correlated adjustments in other developmental processes.

Diversity of gastrulation and cleavage patterns are mirrored by diversity of genetic interactions at the molecular level. Comparisons of well-characterized taxa (insects versus vertebrates) show that gene networks underlying early development allow for substantial molecular reorganization (Holland, 2000; Damen et al., 2000; Stauber et al., 2001). However, evolutionary conservation is probably also important (de Robertís et al., 1994). Holland (2000) concludes, for example, that there are similarities in gene expression during protostome and deuterostome gastrulation, but it is not clear to what extent they arise from actual conservation as opposed tos convergence.

The larger number of cells during gastrulation, increased levels of differentiation, and large permutations of interacting processes generate near infinite variation in gastrulation patterns. Yet, paradoxically, the process of gastrulation is far more diverse than the final end product, gastrulae invariably have two or three germ layers and never more (Hall, 1999). Organ systems emerging from germ layers are similarly conserved; skin and nervous system from ectoderm; digestive tube from endoderm. A key outcome of the process of gastrulation is that sheets of cells come into contact, thus enabling embryonic inductions; neurulation is a classic example. The infinite diversity of subsequent organic forms arguably begins with such inductions of tissue layers and organ systems. However, in order to develop organ systems, inductive events require topologically adjacent cell populations, a requirement that seems to form a stringent spatio-temporal constraint on the outcome of gastrulation.

\section{Conservation, convergence and divergence in early organogenesis}

After germ layers are formed during gastrulation, cells interact with one another and rearrange themselves during organogenesis. This stage among metazoans is far more diverse than earlier stages. However, as mentioned before, within most high order taxa, early organogenesis is far less variable than earlier stages (Seidel, 1960; Sander, 1983; Hall, 1996; Elinson, 1987). Examples of great similarity of post-gastrulation stages occur during the segmented germ-band stage of insects, the nauplius stage of crustaceans, and the neurula/pharyngula stage of vertebrates (Figs 3 and 4; Sander, 1983; Grbi et al., 1998; Dahms et al., 2000). Recent data on genetic interactions confirm that within-taxa similarity is highest shortly after gastrulation (Damen, 2000, Holland, 2000). For example, expression patterns of segment-polarity and Hox genes in insects are more similar to each other than those of earlier acting genes.

The phylogenetic distribution of these patterns of early stages of organogenesis suggests that similarity is due to conservation and not convergence (Damen, 2000). Sander (1983) introduced the term phylotypic stage, as these stages can be seen as typical for the phylum or higher taxa to which they belong. Although phylotypic stages are more conserved than earlier or later developmental stages, we are not suggesting that there is not important variation (see for example Minelli and Schram; 1994 Richardson, 1999); rather, what matters is that there is less variation than at earlier and later stages. Apparently developmental stages evolve at different rates, and constraints to evolutionary change are particularly strong at stages shortly after gastrulation when axis patterning occurs. 


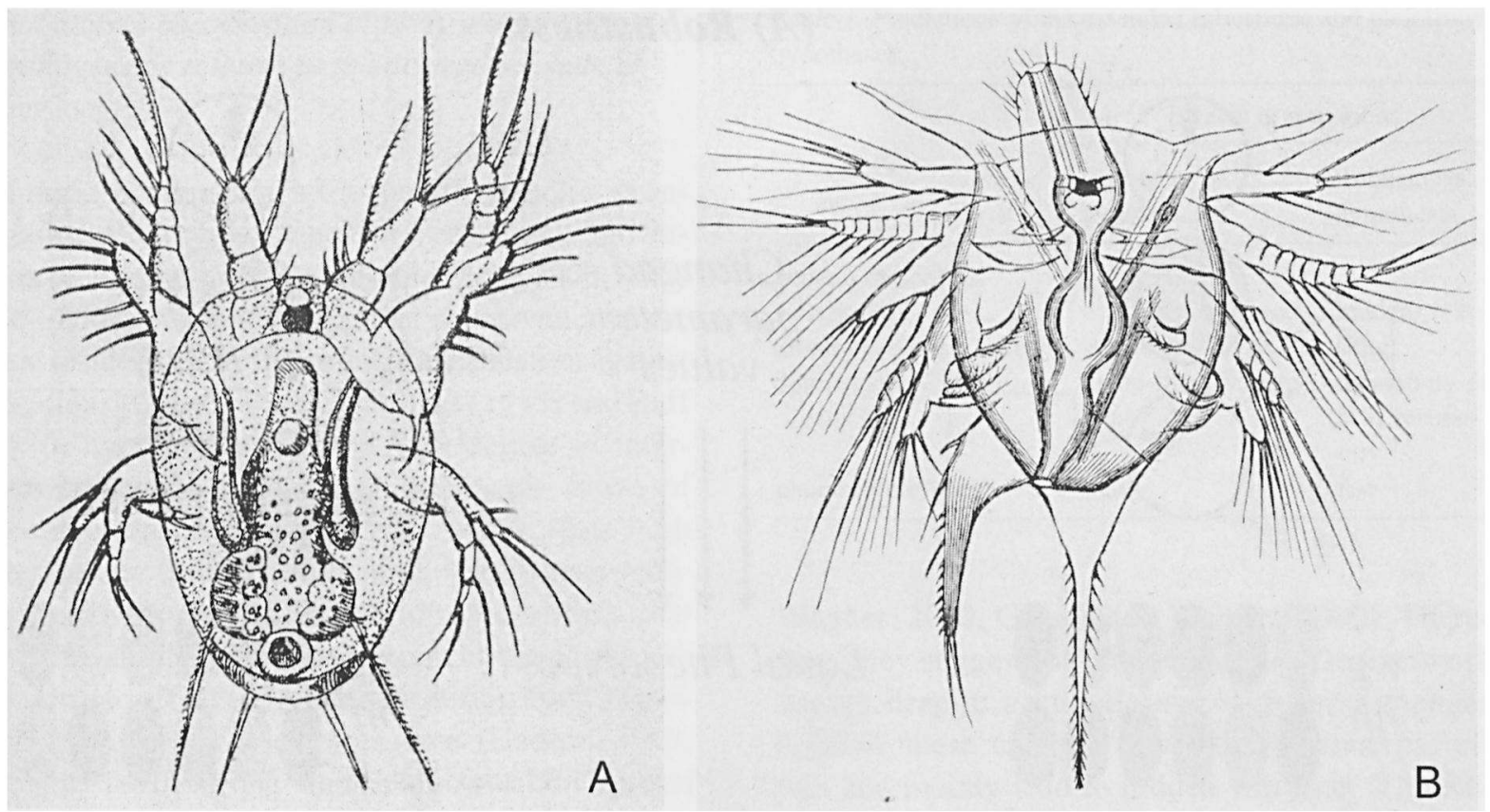

Fig. 3. (A) Early organogenesis stages are very similar in crustaceans. Nauplius stages in (A) Cyclops (from Dawydoff 1928). (B) Cirripedia species (from Claus and Grobben, 1917).

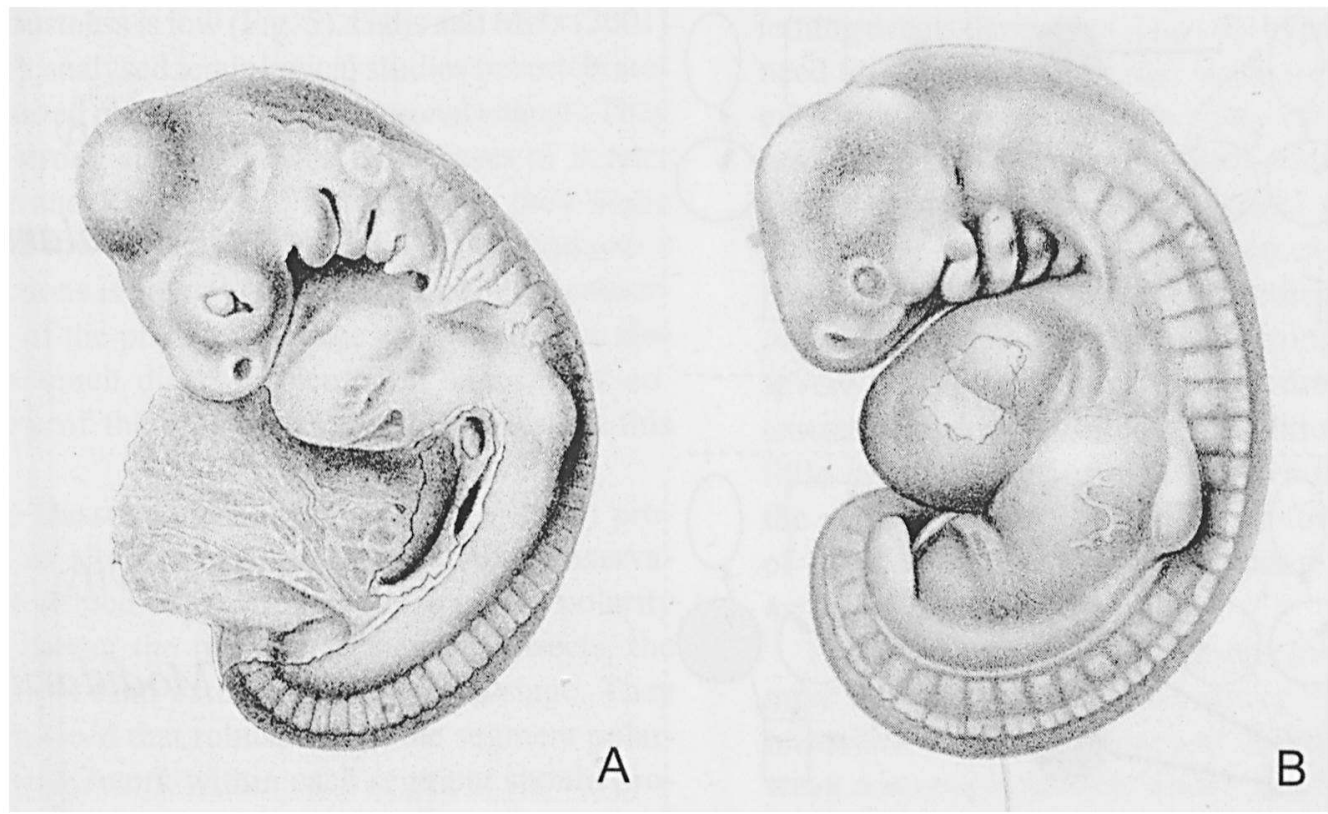

Fig. 4. Early organogenesis stages in vertebrates are more similar than earlier or later stages. Especially within amniotes the similarity is striking. Pharyngula stage in (A) Lacerta and (B) human (from Keibel 1904 and 1908). 


\section{(A) Robustness}
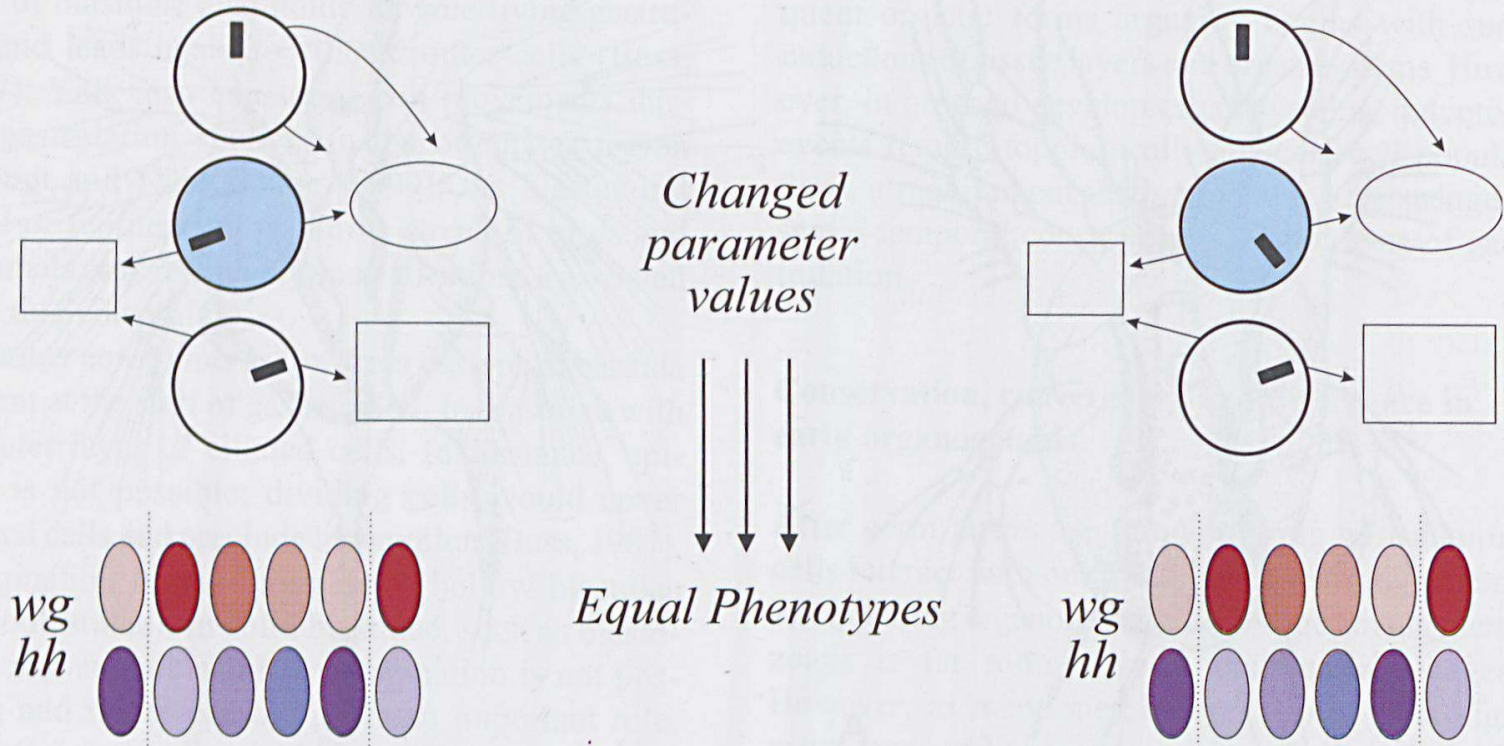

Equal Phenotypes

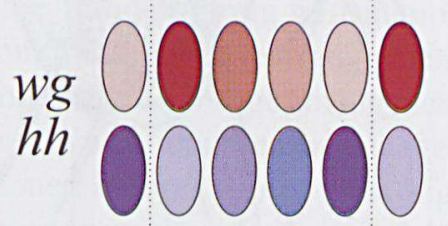

\section{(B) Interacting Modules}

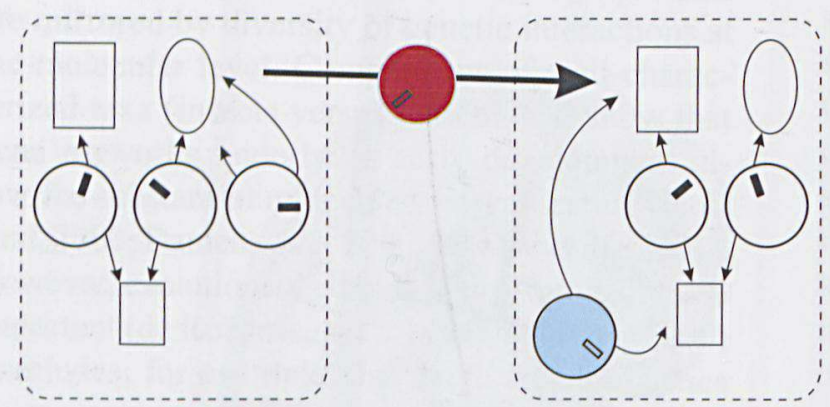

Low Connectivity $=$

High Effective Modularity

\section{High Connectivity} $=$

Low Effective Modularity

Fig. 5. Schematic figure explaining (A) robustness and (B) effective modularity. (A) When a parameter is changed in a robust genetic network, the resulting phenotype does not change (in this case illustrated with the concentration of wingless (wg) and hedgehog (hh) in the cells of the ectoderm). (B) Modules are discernible and discrete units within large genetic networks that have some autonomy and a clear physical location (Raff 1996). They can differ in the amount of connectedness. First of all, all input to robust elements of a module can be ignored, since it will have no discernible effect. A large proportion of robust components in a module therefore reduces potential connectivity. Low connectivity, with few connections having small effects, implies high effective modularity. High connectivity implies low effective modularity. From Galis et al. 2002 
What causes conservation of early stages of organogenesis relative to the divergence seen at other stages?

As there is abundant intra-specific genetic variation for phylotypic stages of vertebrates and insects (Galis et al., 2001, Galis and Metz, 2000, Galis et al., 2002), it would appear that conservation must, thus, result from strong stabilizing selection against mutations (Causes 1 and 2). Sander (1983) and Raff (1996) hypothesize that the high degree of interaction between modules is the major cause of conservation in the phylotypic stages. This high interactivity (low effective modularity) causes mutations to have multiple pleiotropic effects that become amplified as development proceeds. As pleiotropic effects of mutations during embryogenesis are generally disadvantageous (Hadorn, 1961; Wright, 1970) strong stabilizing selection against mutations ensues. In this scenario, conservation is due to consistently strong selection against mutations via their pleiotropic effects. The high interactivity between modules implies an easily destabilized network of inductive events. Therefore, the effective robustness is low (Fig. 5). Galis and Metz (2001) recently analysed teratological studies in vertebrates for reported phenocopies of mutational change. They found strong support for the hypotheses of Sander (1983) and Raff (1996). Furthermore, they argue that the low effective modularity in the inductive interactions is not only the root cause for the conservation of the phylotypic stage as a whole, but also for the much discussed temporal and spatial colinearity of the Hox genes that characterizes this stage.

Von Dassow and colleagues $(1999,2000)$ proposed an alternative hypothesis for the conservation of striped expression of the segment polarity genes during the phylotypic stage in insects, the segmented (and extended) germ band stage. They hypothesized that robustness of the segment polarity gene network within each segment should provide a buffer against phenotypic effects of mutational changes. In robust gene networks, by definition, developmental noise and mutations do not lead to clear phenotypic effects because gene interactions tend to neutralize perturbations and in particular make mutations recessive (Kacser and Burns, 1981;
Table 1. Predictions of the extended robustness and pleiotropy hypotheses.

\begin{tabular}{|c|c|c|}
\hline & \multicolumn{2}{|c|}{ Effects of mutations } \\
\hline & $\begin{array}{l}\text { Pleiotropy } \\
\text { hypothesis }\end{array}$ & $\begin{array}{l}\text { Robustness } \\
\text { hypothesis }\end{array}$ \\
\hline $\begin{array}{l}\text { genetic mutational } \\
\text { variation } \\
\text { direct phenotypic effects } \\
\text { dominance of direct } \\
\text { effects }\end{array}$ & $\begin{array}{l}\text { visible at the } \\
\text { phenotypic level } \\
\text { potentially large } \\
\text { haplo-insufficiency } \\
\text { possible }\end{array}$ & $\begin{array}{l}\text { hidden } \\
\text { small } \\
\text { recessivity or } \\
\text { near recessi- } \\
\text { vity }\end{array}$ \\
\hline pleiotropic effects & many & few \\
\hline
\end{tabular}

Wagner, 2000; Gibson and Wagner, 2000). Therefore, they suggest that conservation of the network occurs despite accumulation of genetic changes because these changes have little phenotypic effect and mainly lead to hidden variation. The Robustness Hypothesis was only proposed for the conservation of the segment polarity gene network during the phylotypic stage. However, this stage as a whole is conserved, and as the segment polarity genes act as an organizer central to most patterning events during this stage, the hypothesis would need to be extended in order to have the presumed evolutionary consequences. The Pleiotropy Hypothesis and Robustness Hypothesis lead to very different predictions for mutations affecting the phylotypic stage (table 1). When evaluating the empirical evidence for these hypotheses in Drosophila, Galis et al. (2002) found strong support for severe phenotypic effects of mutations, including cascading pleiotropic effects. In addition, they found little evidence supporting effective robustness of the segment polarity network, or for interactions of the Hox genes or the organization of the stage as a whole.

There are probably two reasons for the discrepancy between the predicted strong robustness for mutations by von Dassow and colleagues and the weak robustness actually found. First, the organizing function of segment polarity genes causes mutations in these genes to have a cascade of pleiotropic effects with many auto-regulatory and cross-regulatory interactions that provide a feedback on the input of the segment polarity gene network. This leads to a relatively low effective modularity and 
robustness. This feedback that modulates the input of the network is absent in the model of von Dassow et al. Second, concentration differences of gene products appear to have a crucial impact upon morphogenetic patterning, which leads to a high sensitivity of the system to changes. Why has more robustness not evolved? Perhaps, within these stages the total number of interactions involved in morphogenetic patterning is too limited to organize the pattern in an independent modular way to allow greater robustness. The vulnerability to mutations of the most conserved stage in vertebrates, thus, appears to be shared by the most conserved stage in insects. This points to an important role of pleiotropy and stabilizing selection in evolutionary conservation.

Why are these early stages of organogenesis more conserved than earlier and later stages? Earlier stages are simpler in form and for this reason mutations will probably have more of a chance to be successful, since it is more difficult to destabilize a simple pattern than a more complicated pattern. Later stages of organogenesis involve a higher number of inductive interactions than during the phylotypic stage, which presumably allows a higher degree of modularity. A high modularity implies that the effects of mutations will mainly be limited to the module itself and not to other parts of the organism, which in turn increases the "evolvability" of the component parts.

Let us examine an example of a mechanism that promotes divergence after the phylotypic stage via modularity, the role of modular endocrine regulation in promoting divergence. Furthermore we can then perhaps see how divergence of late ontogenetic stages is strongly influenced by the divergence of early ontogenetíc stages.

\section{Modularity and the divergence of form in late organogenesis}

The endocrine control of late organogenesis is formatted into modules and allows for considerable potential to evolve. A classic example involves thyroxine regulation of amphibian metamorphosis during larval development, which has become uncoupled from the development of reproductive structures of the adult. The hypothalamic-pituitary- thyroid (HPT) axis governs metamorphosis (Hayes, $1997 \mathrm{a}, \mathrm{b})$ and is developmentally uncoupled from the hypothalamic-pituitary-gonadal (HPG) axis, which governs sexual maturation. This uncoupling has allowed for the evolution of many heterochronic changes, including paedomorphic lineages in urodeles, e.g., Sirenidae, Amphimidae, Proteidae, Cryptobranchidae Gould, 1977). The uncoupling has further allowed the evolution of alternative feeding morphologies in larval anurans.

In urodeles, deletion of thyroxine regulation has led to a loss of the terrestrial adult phase and paedomorphic larval forms in which sexual maturation occurs in the juvenile form (Gould, 1977). A simple loss-of-function mutation in an endocrine gene of major effect governing thyroxine regulation is responsible for the evolutionary transition of the axolotl, Ambystoma mexicanum, which is derived from the ancestral tiger salamander, $A$. tigrinum, which retains larval metamorphosis (Voss and Shaffer, 1997). The deleterious pleiotropic effects of such mutations are presumably small because of modularity of the endocrine system. Such modularity allows major potential to evolve.

High thyroxine titer does not always induce an aquatic-terrestrial metamorphosis because tissuespecific receptors and other endocrine axes modulate the tissue-specificity of thyroxine. For example, prolactin promotes growth under low "stress", but under stress, e.g., if the water level drops, the hypothalamic-pituitary-adrenal (HPA) axis can rapidly trigger metamorphosis (Hayes, 1997a,b; Denver, 2000). A beautiful example at a fine scale of modularity provides the thyroxine-induction of anuran larval feeding structures (Hanken, 1988). In spadefoot toad tadpoles, Scaphiopus spp., thyroxine ingestion induces a precocious transformation of structures associated with the cranium into more adult-like ones (Pfennig, 1992). Tadpoles in this way adopt a carnivorous form if they ingest shrimps (which contain high levels of thyroxine (Pfennig, $1990,1992)$. This precocious response to thyroxine has evolved many times in taxa with carnivorous larvae and finds an extreme morphological expression in species that have evolved cannibalism (Hanken, 1993; Pfennig and Collins, 1993). 


\section{The relationship between divergence of early and late ontogenetic stages}

The processes that lead to the evolution of large yolk-rich eggs arises apparently mainly from direct selection on later stages of development. Egg size and yolk content are the result of maternal effects and can increase the course of evolution to a threshold size where the feeding larval form can be lost and a non-feeding larval form evolves (Vance, 1973; Raff, 1996). Divergence of early stages, thus, facilitates the divergence of later stages (see also Sinervo and McEdward, 1988). The evolution of a non-feeding larva from a feeding larva further facilitates the evolution of direct development allowing even more divergence. Direct development has evolved many times independently in diverse taxa. The following example of plethodontid salamanders shows how direct development can lead to further specialization and divergence.

Plethodontid salamanders undergo direct development in large yolky eggs and the juveniles hatch into a miniature adult form. During metamorphosis in most salamander families, hyobranchial elements that form larval gills are transformed into the tongue projection feeding apparatus of terrestrial adults. In contrast, in plethodontids where aquatic larval function was lost during the evolution of direct development, hyobranchial structures develop directly for adult function. In addition, the group evolved a lungless condition, respiring through the skin and buccal cavity. Freedom from both feeding and respiratory constraints allowed elaborate tongue projection mechanisms to evolve in some plethodontid salamanders as is seen in the bolitoglossines (see Wake and Larson, 1987). This example, thus, illustrates how the evolved maternal effects on egg size have led to a breaking of functional constraints on development and form thereby allowing novelty to arise. Interestingly, at the same time new constraints arose (yolk that impedes cell division) promoting evolutionary conservation of earlier ontogenetic stages.

The evolution of vivipary in amniotes via egg retention (Laurin et al. 2000) provides another example of how divergence in early ontogenetic stages allows diversification of later ontogenetic forms by allowing niches to be filled that cannot be filled by egg-laying animals.

\section{Early diversification followed by stasis of early stages of organogenesis}

The diversity of early stages of organogenesis among phyla and the similarity within several phyla and other higher order taxa suggests an early phase of rapid diversification in the evolution of metazoans, followed by evolutionary stasis of these discrete taxon-specific stages. Buss (1987) has proposed the following explanation for this phenomenon. The early rapid diversification has happened during the early chaotic phase in the evolution from unicellular to multicellular individuals (presumably during the Cambrian explosion). During the evolution of individuality the level of selection has shifted from individual cells to that of the individual. Early during this transition, somatic mutations in cells that could gain access to the reproductive cells had a chance to be maintained in future generations (as occurred in plants). Later, when selection was firmly established at the level of the individual, heritable mutations were limited to those that happen both in the germ-line, and in the short period before germline sequestration. This scenario is intuitively appealing, but surprisingly little research has yet been carried out to further investigate this important question in evolutionary biology. Mutagenesis experiments with simple colonial organisms and theoretical modeling could probably contribute to a better understanding.

\section{Conclusion}

Rapid progress that has been made in understanding genetic and morphogenetic patterning of several invertebrate and vertebrate model species allows us to determine in many cases whether similarity arises from conservation or convergence. Unfortunately, for many invertebrate taxa, we lack crucial information that allows for meaningful interpretations. Emergent patterns suggest that at least two phenomena are responsible for the phenomenon that overall similarity within metazoans is greatest during the early embryonic stages of cleavage and gastrulation, whereas yet in many taxa early organogenesis is more similar and conserved. First, the similarity of cleavage and gastrulation is not only 
due to conserved similarity, but also to an important extent due to convergence. Second, the phylogenetic pattern of early organogenesis implies that there has been an early phase of diversification in the evolution of metazoans, followed by extreme stasis of these discrete taxon-specific stages. An important cause of conservation appears to be stabilizing selection against negative pleiotropic effects. The causes for the initial evolutionary divergence of taxa are not clear and remain a challenge for future evolutionary developmental research, as do the diverse forms of uninvestigated taxa of invertebrates.

\section{Acknowledgements}

We thank Jacques van Alphen, Ricardo Azevedo, Ronald Jenner, Hans Metz, Urs Schmidt-Ott, and Frederick Schram for insightful comments on the manuscript and Joris van Alphen, Peter van Mulken, and Fineke Speksnijder for help with the figures.

\section{References}

Anderson DT. 1973. Embrology and Phylogeny in annelids and Arthropods. Oxford: Pergamon.

Arendt D, Nübler-Jung K. 1999, Rearranging gastrulation in the name of yolk: evolution of gastrulation in yolk-rich amniote eggs. Mechanisms of Development 81: 3-22.

Baer KE.von 1828. Über Entwickelungsgeschichte der Thiere: Beobachtung und Reflexion. Erster Theil. Königsberg, xxï+271.

Balfour FM. 1881. Comparative embryology. London: Macmillan and Co.

Buss LW. 1987. The evolution of individuality. Princeton: Princeton University Press.

Claus C, Grobben K. 1917. Lehrbuch der Zoologie. Marburg: Elwert'sche Verlagsbuchhandlung.

Collazo A, Bolker JA, Keller R. 1994. A phylogenetic perspective on teleost gastrulation. Amer. Natur. 144: 133-152.

Dahm H-U. 2000. Phylogenetic implications of the crustacean nauplius. Hydrobiology 417: 91-99.

Damen WGM, Weller M, Tautz D. 2000. Expression patterns of hairy, even-skipped, and runt in the spider Cupiennius salei imply that these genes were segmentation genes in a basal arthropod. Proc. Nat, Acad. Sci, USA, 97: 4515-4519.

Dawydoff C. 1928. Traité d'embryologie comparée des invertébrés. Paris : Masson et Cie.

Denver RJ. 2000. Evolution of the cotticotropin-releasing hormone signaling and its role in stress-induced developmental plasticity. Amer, Zool. 40: 995-996.

De Robertis EM, Fainsod A, Gont LK, Steinbeisser H. 1994. The evolution of invertebrate gastrulation. Development (Suppl.), 1994: 117-124.
Elinson RP. 1987. Change in developmental patterns: Embryos of Amphibians with large eggs. In: RA. Raff and EC. Raff (eds). Development as an Evolutionary Process pp. 1-21. Alan R. Liss, Inc.

Galis F, Metz JAJ. 2001. Testing the vulnerability of the phylotypic stage: on modularity and evolutionary conservation. J. Exper. Zool. 291: 195-204.

Galis F, van Alphen JAJ, Metz JAJ. 2001. Why five fingers? Evolutionary constraints on digit numbers. Trend Ecol. Evol. 16: 637-646.

Galis F, van Dooren TJM, Metz JAJ. 2002. Conservation of the segmented germband stage: robustness or pleiotropy? Trends Genet. 18 (10):504-509.

Gibson G, Wagner GP. 2000. Canalization in evolutionary genetics: a stabilizing theory? BioEssays $22: 372-380$.

Gilbert SF, 2000. Developmental Biology. Sixth ed. Sinauer Associates, Sunderland, MA.

Gilbert SF, Raunio AM. 1997. Embryology. Constructing the organism. Sinauer Associates, Sunderland, MA.

Gould SJ. 1977. Ontogeny and Phylogeny, Cambridge, MA Belknap Press, Havard Univ. Press.

Grbič, M, Nagy LM, Stran MR. 1998. Development of polyembryonic insects: a major departure from typical insect embryogenesis. Develop. Genes Evol. 208: 69-81.

Hadorn E. 1961. Developmental genetics and lethal factors. Methuen and $\mathrm{Co}$, London.

Hall BK. 1 996. Baupläne, phylotypic stages, and constraìnt. Why are there so few types of animals? Evol. Biol. 29: 215261.

Hall BK. 1999. Evolutionary Developmental Biology. Second Edition. Kluwer Academic Publishers, Dordrecht, Netherlands.

Hanken J. 1988. Skull development during anuran metamorphosis: 3. Role of thyroid-hormone in chondrogenesis. $J$ Lxp. Zool. 246: 156-170.

Hanken J. 1993. Model systems versus outgroups - alternative approaches to the study of head development and evolution. Amer, Zool. 33: 448-456.

Hayes TB. 1997a. Steroids as potential modulators of thyroid hormone activity in anuran metamorphosis. Amer, Zool. 37: 185-194.

Hayes TB. 1997b. Hormonal mechanisms as potential constraints on evolution: examples from the Anura, Amer, Zool. 37: $482-490$.

Holland LZ. 2000. Body-plan evolution in the Bilateria: early antero-posterior patterning and the deuterostome-protostome dichotomy. Current Opinion in Genetics and Development 10: 434-442.

Kacser H. Burns JA, 1981. The molecular basis of dominance. Genetics 97: 639-666.

Keibel F. 1904. Normentafeln zur Entwicklungsgeschichte der Wirbeltiere, Heft IV, Gustav Fisher, Jena.

Keibel F, 1908. Normentafeln zur Entwicklungsgeschichte der Wirbeltiere, Heft VIII, Gustav Fisher, Jena

Laurin M, Reisz RR, Girondot M. 2000. Caecilian viviparity and amniote origins: a reply to Wilkinson and Nussbaum. $J$. Nat. Hist. 34: 311-315. 
Minneli A, Schram FR. 1994. Owen revisited; a reappraisal of morphology in evolutionary biology. Bijdr. Dierkunde 64: 65-74.

Pfennig DW. 1992. Proximate and functional causes of polyphenism in an anurań tadpole. Func. Ecol. 6: 167-174.

Pfennig DW, Collins JP. 1993. Kinship affects morphogenesis in cannibalistic salamanders. Nature 362: 836-838.

Raff RA. 1996. The shape of life. The University of Chicago Press, Chicago.

Richardson MK. 1999. Vertebrate evolution. The developmental origins of adult variation. Bioessays 21: 604-613.

Sander K. 1983. The evolution of patterning mechanisms: Gleanings from insect embryogenesis and spermatogenesis. In : Goodwin, B.C., N. Holder, and C.C. Wylie, eds. Development and Evolution. pp. 137-160, Cambridge University Press, Cambridge.

Seídel F. 1960. Körpergrundgestalt und Keimstruktur. Eìne Erörterung über die Grundlagen der vergleichenden und experimentellen Embryologie und deren Gültigkeit beì phylogenetischen Berlegungen. ZooL. Anz. 164: 245-305.

Sinervo B, McEdward LR. 1988. Developmental consequences of an evolutionary change in egg size: an experimental test. Evolution 42: 885-899.
Slack JMW, Holland PWH, Graham CF. 1993. The zootype and the phylotypic stage. Nature 361: 490-492.

Stauber M, Prell A, Schmidt-Ott U. 2001. A single Hox3 gene with composite bicoid and zerknüllt expression characteristies in non-cyclorrhaphan flies. Proc. Nat. Acad. Sci., USA, in press.

Vance RR. 1973. On reproductive strategies in marìne benthic invertebrates. Amer, Natur. 107: 339-352.

Viebahn C. 1999. The anterior margin of the mammalian gastrula: Comparative and phylogenetic aspects of its role in

- axis formation and head induction. Current Topics Devel. Biol. 46: 64-103.

Voss SR, Shaffer HB. 1997. Adaptive evolution vía a major gene effect: Paedomorphosis in the Mexican axolotl. Proc. Natl. Acad. Sci. USA 94: 14185-14189.

Wagner A. 2000. Robustness against mutations in genetic networks of yeast. Nature Genet. 24: 355-361.

Wake DB, Larson A. 1987. Multidimensional analysis of an evolving lineage. Science 238: 42-48.

Wright TR. 1970. The genetics of embryogenesis in Drosophila. Adv. Genet. 15: 261-395.

Accepted: 16 September 2002 